\title{
Performance of Two Sample Scale Tests: An Empirical Study
}

\author{
Pronita Gogoi ${ }^{1}$ and Bipin Gogoi ${ }^{2}$ \\ Research Scholar, Dept of Statistics, Dibrugarh University ${ }^{1}$ \\ Professor, Dept of Statistics, Dibrugarh University ${ }^{2}$
}

\begin{abstract}
For the two samples scale problem, we have considered the ten existing tests viz. F-test, Mood test, AnsariBradley test, Siegel-Tukey test, Lepage type test, Cramer-Von-Mises test, Kamat test, Wald-W test, Wald-R test and Levene test. To study the performance of these tests we generate data from the distributions viz. Normal, Exponential, Double Exponential, Logistic and Cauchy. We have calculated the empirical level under null situations and power under alternative situations taking various combinations of scale parameters and sample sizes using simulation technique. We also simulated critical values for Kamat test, Wald-W test and Wald-R test for some sample sizes. Results are displayed in various tables and graphs. Discussion and conclusion are made on the basis of results obtained.
\end{abstract}

Keywords: Mood test, Ansari-Bradley test, Siegel-Tukey test, Lepage type Test, Cramer -von Mises test, Kamat test, Wald-W test, Wald-R test, Levene test, Simulation study, Symmetrical and Asymmetrical Distributions.

\section{INTRODUCTION}

The study of variance difference differences in two or more populations is one of the important problem in statistical inference. There are many parametric, nonparametric tests are developed till now. In the early years, Lehmann (1951), Mood (1954) and Shukhatme (1957) developed some nonparametric tests of variance differences. The procedure suggested by Lehmann however, has been shown not to be distribution free. The test proposed by Mood assumes knowledge of the means of the two populations, whereas in practice such knowledge rarely exists. The procedure suggested by Shukhatme also assumes knowledge regarding the location of the two populations and in addition this procedure is not as efficient as the one proposed by Mood. Procedures proposed by Kamat (1956) and Rosenbaum (1953) seem likely to lack power. Ansari and Bradley (1960) developed a rank test for dispersion. They have shown the equivalence of two rank tests for comparing dispersion, one test due to Barton and David (1958), the other to Ansari and Freund, and have provided tables of the exact distribution. They observed that Siegel and Tukey (1960) have proposed a similar test which permits use of existing tables. They also exhibit the mean of the limiting normal distribution under the alternative hypothesis .Assuming normality of the data the $\mathrm{t}$ test and $\mathrm{F}$ test are uniformly most powerful unbiased test in the case of location and scale alternatives respectively, and it is well known that the $t$ test is $\alpha$-robust for non normal distributions, expect for those with very long tails, but not $\beta$ - robust, whereas $F$ test is extremely not $\alpha$ robust for non normal data. Perng Littell (1976) proposed a test Q of equality of means and variances in the case of normal population where $Q$ is mixture of $\mathrm{t}$ test and $\mathrm{F}$ test. As the $\mathrm{F}$ test $\mathrm{Q}$ test, however is not $\alpha$ robust for non normal data. So in this connection Buning and Thadewald (2000) looked for tests which are generally powerful for non normal data and for location and scale alternative. One of the most powerful tests for this problem is the test of Lepage, which is a combination of the Wilcoxon test for location and Ansari Bradley test for scale. For General alternatives including different shapes of distributions of the $\mathrm{X}$ and $\mathrm{Y}$ variables the tests of Kolmogorov - Smirnov, Cramer - Von Mises and WaldWolfowitz might be the most popular ones, where CramerVon-Mises test seems to be the best one among these non parametric tests over a broad class of distributions. Hall and Padmanabhan (1997) have proposed some tests, which are Flinger - Killeen ( $\mathrm{F}-\mathrm{K}$ : med) type modification of the Ansari-Bradley, Mood and Klotz test and estimated their quantities by means of the smooth bootstrap. In 2011, Padmanabhan, Othman and Yin proposed variants of the above tests, called refined robust test or refinements, for short and showed that they are superior to the standard test and in addition their performance improves with increasing sample sizes.

Allingham and Rayner(2011) introduced a new test for equality of variances based on the nonparametric analogue of a very natural Wald test and call it the R Test. In an indicative empirical study they showed that, for moderate sample sizes when assuming normality the R test is nearly as powerful as the F test and nearly as robust as Levene's test. It is an appropriate test for equality of variances without the assumption of normality, and so it can be strongly recommended.

Our aim of study is to compare the ten existing tests viz.Ftest Mood test, Ansari-Bradley test, Siegel-Tukey test, Lepage Type Test (Lepage Test), Cramer-von-Mises test, Kamat test, Wald-W test, Wald-R test and Levene test for testing difference of scale parameters. We here use Monte Carlo simulation technique for find the results by generating observations for symmetric and asymmetric 
distributions (normal, exponential, double exponential, associated with each sample should be approximately logistic and cauchy). In this paper as we observed that equal. On the other hand, if the score spread is not Wald-W test is not able to satisfy the specified homogeneous for the two groups, then the rank sum of the significance level under Cauchy, exponential and double sample with the greatest spread will be significantly exponential distributions and so we are not calculating smaller than the rank sum of the more compressed sample. power of this test under these distributions. In section 2 we Using an indicator variable, $Z_{i}$, let $Z_{i}=1$ if the $i^{\text {th }}$ describe the test statistics and section 3 contains some replacement score is associated with the $\mathrm{X}$ sample and simulation power of the tests considered. Discussion and $\mathrm{Z}_{\mathrm{i}}=0$ if the $\mathrm{i}^{\text {th }}$ score is tagged to the $\mathrm{Y}$ sample. This conclusion are given in section 4 and section 5. The indicator variable is useful in setting up the test statistic simulated critical values of Wald-W test and Wald $-\mathrm{R}$ test which is defined as have shown in Table - 3

\section{TEST STATISTICS}

$$
\mathrm{ST}=\sum_{\mathrm{i}=1}^{\mathrm{N}} \mathrm{iZ}
$$

Let $X_{1}, X, \ldots, X_{m}$ and $Y_{1}, Y_{2}, \ldots, Y_{n}$ be two independent random sample from population with absolutely continuous distribution function $\mathrm{F}\left(\frac{\mathrm{X}-\mu_{1}}{\sigma_{1}}\right) \operatorname{andF}\left(\frac{\mathrm{Y}-\mu_{2}}{\sigma_{2}}\right)$, respectively. We wish to test the null hypothesis $\mathrm{H}_{0}$ : $\sigma_{1}=\sigma_{2}, \quad \mu_{1}=\mu_{2} \quad$ Vs $H_{1}: \sigma_{1}>\sigma_{2}, \quad \mu_{1}=\mu_{2}$

\section{Mood Test (M):}

In closer analogy with classical test for scale, Mood has proposed a test using as test statistic

$$
\mathrm{M}=\sum_{\mathrm{i}=1}^{\mathrm{n}}\left(\mathrm{R}_{\mathrm{i}}-\frac{\mathrm{n}+\mathrm{m}+1}{2}\right)^{2}
$$

Where $R_{i}$ is the size rank of $X_{i}$ in the pooled sample (the smallest of whose observations receives a rank of 1 , the next smallest a 2, etc., and largest a rank of $n+m)$ and ( $+m+1) / 2$ is the average rank of all observations comprising the pooled sample. The null distribution of $\mathrm{M}$ has a mean of $[\mathrm{n}(\mathrm{n}+\mathrm{m}+1)(\mathrm{n}+\mathrm{m}-1)] / 12$ and a variance of $[n m(n+m+1)(n+m+2)(n+m-2)] / 180$, and the test apparently consists of treating $\mathrm{M}$ as a normal deviate.

\section{Siegel Tukey Test (ST):}

This test replaces the pooled data from the two sample with a reordering of the ranks (i) from 1 to N. to illustrate the ranking procedure, note the following table when $\mathrm{N}$ is

\begin{tabular}{|c|c|c|c|}
\hline $\begin{array}{l}\text { Ordered Score } \\
\qquad V^{N / 2} \ldots \ldots V^{N-3}\end{array}$ & $\begin{array}{l}\mathrm{V}^{1} \\
\mathrm{~V}^{\mathrm{N}-2}\end{array}$ & $\begin{array}{l}\mathrm{V}^{2} \\
\mathrm{~V}^{\mathrm{N}-1}\end{array}$ & $\begin{array}{c}\mathrm{V}^{3} \ldots \ldots \\
\mathrm{V}^{\mathrm{N}}\end{array}$ \\
\hline Rank Replacement & 1 & 4 & $5 \ldots \ldots$ \\
\hline $\begin{array}{ll}\ldots \ldots 7 & 6\end{array}$ & 2 & & \\
\hline
\end{tabular}
assumed to be an even number

At the left end of the ordered set of scores, $\mathrm{V}^{1}$ is assigned a rank of 1 . The test development now requires a move to the extreme right end of the ordered scores where $\mathrm{V}^{\mathrm{N}}$ is replaced by the rank 2 and $\mathrm{V}^{\mathrm{N}-1}$ by the rank 3 . Now move back to the left and substituted the ranks 4 for $\mathrm{V}^{2}$ and 5 for $\mathrm{V}^{3}$. Operating the pairs, this process is repeated until all ordered scores are replaced by their appropriate ranks. If $\mathrm{N}$ is an odd number, throw out the middle score. This will enable the adjacent ranks to sum to the same number and thus achieve a desired symmetry to the test. If there is no difference in scale between the populations from which the two samples are drawn, then the sum of the ranks
The null distribution of the ST test is exactly the same as that of the Wilcoxon test. Thus, Wilcoxon table can be used to determine the significance of ST forN $<20$. Equivalent table have been developed by Siegel and Tukey (1960). For $\mathrm{N}<20$ the distribution of ST approximates a normal distribution with $\mathrm{E}(\mathrm{ST})=$ $\mathrm{n}(\mathrm{N}+1) / 2 \quad \operatorname{andVar}(\mathrm{ST})=\mathrm{nm}(\mathrm{N}+1) / 12$. The Test statistics become

$$
\mathrm{Z}=\frac{(\mathrm{ST})-\mathrm{E}(\mathrm{ST})}{\sqrt{\operatorname{Var}(\mathrm{ST})}}
$$

where $\mathrm{Z}$ follows standard normal distribution.

\section{Cramer - Von Mises Test (CVM):}

Let $X_{(1)}, X_{(2)}, \ldots, X_{(m)}$ and $Y_{(1)}, Y_{(2)}, \ldots, Y_{(n)}$ be the order statistics of $X_{1}, X_{2}, \ldots, X_{m}$ and $Y_{1}, Y_{2}, \ldots, Y_{n}$ respectively and let $\widetilde{F}_{m}$ and $\widetilde{G}_{n}$ be the usual empirical distribution function for the $\mathrm{X}$ and $\mathrm{Y}$ samples. Furthermore, let be $\mathrm{Z}_{(1)}, \mathrm{Z}_{(2)}, \ldots, \mathrm{Z}_{(\mathrm{N})}$ the order statistics of the combined two samples.

Note that

$$
\begin{aligned}
& \widetilde{F}_{m}\left(Y_{(j)}\right)=\frac{\left[R\left(Y_{(j)}\right)-j\right]}{m}, j=1,2, \ldots, n \\
& \widetilde{G}_{n}\left(X_{(i)}\right)=\frac{\left[R\left(X_{(i)}\right)-i\right]}{n}, i=1,2, \ldots, m
\end{aligned}
$$

Where $\mathrm{R}\left(\mathrm{X}_{(\mathrm{i})}\right)$ and $\mathrm{R}\left(\mathrm{Y}_{(\mathrm{j})}\right)$ are rank of $\mathrm{X}_{(\mathrm{i})}$ and $\mathrm{Y}_{(\mathrm{j})}$ among $\mathrm{Z}_{(1)}, \mathrm{Z}_{(2)}, \ldots \mathrm{Z}_{(\mathrm{N})}$, respectively. The Cramer - Von -Mises statistics $(\mathrm{CM})$ is defined as follows,

$$
\mathrm{CM}=\frac{\mathrm{mn}}{\mathrm{N}^{2}} \sum_{\mathrm{i}=1}^{\mathrm{N}}\left[\widetilde{\mathrm{F}}_{\mathrm{m}}\left(\mathrm{Z}_{(\mathrm{i})}\right)-\widetilde{\mathrm{G}}_{\mathrm{n}}\left(\mathrm{Z}_{(\mathrm{i})}\right)\right]^{2}
$$

$\mathrm{CM}$ can be rewritten as

$$
\mathrm{CM}=\frac{\sum_{\mathrm{i}=1}^{\mathrm{m}}\left[\mathrm{R}\left(\mathrm{X}_{(\mathrm{i})}-\mathrm{i}\right)\right]^{2}}{\mathrm{nN}}+\frac{\sum_{\mathrm{j}=1}^{\mathrm{n}}\left[\mathrm{R}\left(\mathrm{Y}_{(\mathrm{j})}-\mathrm{j}\right)\right]^{2}}{\mathrm{mN}-1}
$$

The Corresponding test reject $\mathrm{H}_{0}$, if $\mathrm{CM} \geq \mathrm{C}_{1-\alpha}(\mathrm{m}, \mathrm{n})$. Exact and asymptotically critical values $\mathrm{C}_{1-\alpha}$ can be found in Burr (1963). 


\section{Lepage Test ( $L)$ :}

The test of Lepage (1971) is a combination of the Wilcoxon test $\mathrm{W}$ for location alternatives and the AnsariBradley test $\mathrm{AB}$ for scale alternatives. The $\mathrm{W}-$ test is locally most powerful for the logistic distribution and $A B$ - test for a special Mielke distribution. The Wilcoxon statistic $\mathrm{W}$ is defiened by

$$
\mathrm{W}=\sum_{\mathrm{i}=1}^{\mathrm{m}} \mathrm{R}\left(\mathrm{X}_{(\mathrm{i})}\right)
$$

where $\mathrm{R}\left(\mathrm{X}_{(\mathrm{i})}\right)$ is the rank of $\mathrm{X}_{(\mathrm{i})}$ in the combined two samples. The Wilcoxon test corresponds to the location model $\mathrm{G}(\mathrm{z})=\mathrm{F}(\mathrm{z}-\theta)$ with the hypothesis $\mathrm{H}_{0}: \theta=0$ versus $\mathrm{H}_{1}: \theta<0, \theta>0$ or $\theta \neq 0$

Under $\mathrm{H}_{0}$, we have $(\mathrm{N}=\mathrm{m}+\mathrm{n})$ :

$$
\begin{gathered}
\mu_{\mathrm{w}}=\mathrm{E}(\mathrm{W})=\frac{\mathrm{m}(\mathrm{N}+1)}{2}, \\
\sigma_{w}^{2}=\operatorname{Var}(W)=\frac{m n(N+1)}{12}
\end{gathered}
$$

The Ansari-Bradley test corresponds to the scale model $\mathrm{G}$ $(\mathrm{z})=\mathrm{F}(\mathrm{z} / \tau)$, where $\tau$ is a scale parameter, with the hypotheses $H_{0}: \tau=1$ versus $\left.H_{1}: \tau<1, \tau\right\rangle 1$ or $\tau \neq$ 1 . The Corresponding test statistics is defined by

$$
\begin{gathered}
A B=\sum_{i=1}^{m}\left((N+1) / 2-\left|R\left(X_{(i)}\right)-(N+1) / 2\right|\right) \\
\mu_{A B}=E(A B)= \begin{cases}\frac{m(N+2)}{4} & \text { if } N \text { is even } \\
\frac{m(N+1)^{2}}{4 N} & \text { if } N \text { is odd }\end{cases} \\
\sigma_{A B}^{2}=\operatorname{Var}(A B) \\
= \begin{cases}\frac{m n\left(N^{2}-4\right)}{48(N-1)} & \text { if } N \text { is even } \\
\frac{m n(N+1)\left(N^{2}+3\right)}{48 N^{2}} & \text { if } N \text { is odd }\end{cases}
\end{gathered}
$$

Then the Statistics LP of Lepage is defined by

$$
L P=\left(\frac{W-\mu_{w}}{\sigma_{w}}\right)^{2}+\left(\frac{A B-\sigma_{A B}}{\sigma_{A B}}\right)^{2}
$$

In Lepage (1971) it is shown that, under $H_{0}$, the statistics $\mathrm{W}$ and $\mathrm{AB}$ are uncorrelated for all $\mathrm{m}, \mathrm{n}$ and as a consequence, the asymptotic distribution of LP is, under $H_{0}, \chi^{2}$ with 2 degree of freedom, that is $H_{0}$ is rejected if $L P \geq \chi_{1-\alpha}^{2}(2)$.

\section{Kamat Test (K):}

Let $R_{n}$ and $R_{m}$ be the range of ranks of $\mathrm{x}$ and $\mathrm{y}$ respectively. The test statistic proposed is

$$
D_{n, m}=R_{n}-R_{m}+m
$$

Where $D_{n, m}$ can take values $0,1,2, \ldots, \mathrm{m}+\mathrm{n}$. Large or small values of $D_{n, m}$ will indicate possible divergence from the hypothesis that the parameters of dispersion of the populations from which the samples have been drawn are equal. Percentage points of $D_{n, m}$ are available in
Kamat (1956). For some values of $m$ and $n$ we have calculated by simulation.

\section{Wald Test:}

A Wald test statistics for testing $H: \theta=\sigma_{2}^{2}-\sigma_{1}^{2}=0$ against $K: \theta \neq 0$ is as follows

$$
W=\frac{\left(S_{1}^{2}-S_{2}^{2}\right)^{2}}{2 S_{1}^{4} /\left(n_{1}+1\right)+2 S_{2}^{4} /\left(n_{2}+1\right)}
$$

Being a Wald test, the asymptotic distribution of $\mathrm{W}$ is $\chi_{1}^{2}$, while its exact distribution is not obvious. However, $\mathrm{W}$ is a one to one function of $F$, and so the two test are equivalent. Since the exact distribution of $\mathrm{F}$ is known, the $\mathrm{F}$ test is the more convenient test.

The variances $\operatorname{var}\left(S_{j}^{2}\right)$ used in $\mathrm{W}$ are estimated optionally using the Rao-Blackwell theorem. This depends very strongly on the assumption of normality. If normality is in doubt then we can estimate $\operatorname{var}\left(S_{1}^{2}-S_{2}^{2}\right)$.

A robust alternative to $\mathrm{W}$ is thus

$$
R=\frac{\left(S_{1}^{2}-S_{2}^{2}\right)^{2}}{\left(m_{14}-S_{1}^{4}\right) / n_{1}+\left(m_{24}-S_{2}^{4}\right) / n_{2}}
$$

In which $m_{i 4}, i=1,2$, are the fourth central sample moments for the $\mathrm{i}^{\text {th }}$ sample, $S^{4}=n m_{2}^{2} /(n-1)$ and $S^{2}$ is the unbiased sample variance, . We call the test based on $\mathrm{R}$ the $\mathrm{R}$ Test. In large samples the denominator in $\mathrm{R}$ will approximate $\operatorname{var}\left(S_{1}^{2}-S_{2}^{2}\right)$ and $\mathrm{R}$ will have asymptotic distribution $\chi_{1}^{2}$.

\section{Levene Test:}

The statistics of Levene is just the square of the $\mathrm{t}$ - statistic applied on transformed X's and y's. Let be transformations $X_{i}^{\prime}=\left|X_{i}-\bar{X}\right| \quad$ and $\quad Y_{i}^{\prime}=\left|Y_{i}-\bar{Y}\right|, \quad i=1, \ldots, m, \quad j=$ $1, \ldots, n$, of the random variables $X_{1}, \ldots, X_{m}$ and $Y_{1}$, $\ldots, Y_{n}$, respectively. Furthermore, let be $\bar{X}^{\prime}$ and $\bar{Y}$ the arithmetic means of the $X_{i}^{\prime}$ and $Y_{j}^{\prime}$ variables. The Levene statistics is then defined by

$$
\begin{aligned}
& L V=\frac{\left(\bar{X}^{\top}-\bar{Y}^{\prime}\right)^{2}}{S^{\prime 2}(1 / m+1 / n)} \text { with the pooled variance } \\
& S^{\prime 2}=\frac{\sum_{i=1}^{m}\left(X_{i}^{\prime}-\overline{X^{\prime}}\right)^{2}+\sum_{j=1}^{n}\left(Y_{i}^{\prime}-\overline{Y^{\prime}}\right)^{2}}{m+n-2}
\end{aligned}
$$

The statistics LV has, under $H_{0}$, approximately a $\mathrm{F}$ distribution with $(1, m+n-2)$ degrees of freedom. Thus, the Levene test reject $H_{0}$ if $L V \geq F_{1-\alpha}(1, m+n-$ 2.

\section{F- Test:}

We assume independent random sample of size $m$ and $n$ from normal population, $N\left(\mu_{i}, \sigma_{i}^{2}\right)$ for $\mathrm{i}=1,2$. We wish to test $H o: \sigma_{1}^{2}=\sigma_{2}^{2}$ against the alternative $K: \sigma_{1}^{2} \neq \sigma_{2}^{2}$. If $S_{i}^{2}, i=1,2$ are the unbiased sample variance, then the so called $\mathrm{F}$ test is equivalent to the likelihood ratio test and is based on the quotient of the sample variance, $\mathrm{S}_{2}^{2} / \mathrm{S}_{1}^{2}=\mathrm{F}$. The null distribution of $F$ namely $F_{m-1, n-1}$ is sensitive to 
Vol. 3, Issue 11, November 2016

departures from normality. If the cumulative distribution null hypothesis of equal variance is studied against the function of this distribution is $\mathrm{F}_{\mathrm{m}-1, n-1}(\mathrm{x})$, and if $\mathrm{c}_{\mathrm{p}}$ is alternatives (introducing scale parameter with different such that $\mathrm{F}_{\mathrm{m}-1, \mathrm{n}-1}\left(\mathrm{c}_{\mathrm{p}}\right)=\mathrm{p}$, then the $\mathrm{F}$ test rejects Ho at values and equal location parameter in $\mathrm{Y}$ population) such the $100_{\alpha \%}$ level when $\mathrm{F} \leq \mathrm{c}_{\alpha / 2}$ and when $\mathrm{F} \geq \mathrm{c}_{1-\alpha / 2}$.

\section{SIMULATION}

To determine the significance level and power of the tests, we generate random sample from the normal, exponential, double exponential, logistic and Cauchy distribution. The as: $(1.5,1),(2,1),(2.5,1)$ and $(3,1)$. Here Table-1(a) to 1(e) gives the estimates of significance level of the considered tests for normal, exponential, double exponential, logistic and Cauchy distribution respectively. Table-2(a) to 2(e) gives the estimates of power of the tests for normal. Exponential, double exponential, logistic and Cauchy distribution respectively.

Table -1(a): Empirical level of tests under Normal Distribution based on 10, 000 replications.

\begin{tabular}{|c|c|c|c|c|c|c|c|c|c|c|}
\hline $\mathrm{n}_{1}, \mathrm{n}_{2}$ & Mood & $\begin{array}{c}\text { Ansari } \\
\text { Bradley }\end{array}$ & $\begin{array}{c}\text { Siegel } \\
\text { Tukey }\end{array}$ & Lepage & CVM & Kamat & $\begin{array}{c}\text { Wald } \\
\mathrm{W}\end{array}$ & $\begin{array}{c}\text { Wald } \\
\mathrm{R}\end{array}$ & Levene & $\mathrm{F}$ \\
\hline \multirow{3}{*}{5,10} & 0.107 & 0.112 & 0.112 & 0.089 & 0.101 & 0.103 & 0.100 & 0.100 & 0.092 & 0.131 \\
& 0.054 & 0.042 & 0.063 & 0.037 & 0.052 & 0.042 & 0.050 & 0.050 & 0.043 & 0.066 \\
& 0.028 & 0.023 & 0.025 & 0.003 & 0.011 & 0.011 & 0.010 & 0.010 & 0.005 & 0.013 \\
\hline \multirow{3}{*}{10,10} & 0.104 & 0.099 & 0.107 & 0.100 & 0.103 & 0.108 & 0.100 & 0.100 & 0.080 & 0.097 \\
& 0.048 & 0.042 & 0.055 & 0.046 & 0.047 & 0.051 & 0.050 & 0.050 & 0.038 & 0.046 \\
& 0.022 & 0.022 & 0.029 & 0.005 & 0.009 & 0.020 & 0.010 & 0.010 & 0.005 & 0.009 \\
\hline \multirow{3}{*}{10,15} & 0.104 & 0.097 & 0.101 & 0.099 & 0.100 & 0.179 & 0.100 & 0.100 & 0.073 & 0.113 \\
& 0.052 & 0.049 & 0.052 & 0.047 & 0.050 & 0.099 & 0.050 & 0.050 & 0.033 & 0.056 \\
& 0.026 & 0.023 & 0.024 & 0.005 & 0.010 & 0.015 & 0.010 & 0.010 & 0.004 & 0.012 \\
\hline \multirow{3}{*}{15,15} & 0.102 & 0.101 & 0.101 & 0.099 & 0.102 & 0.119 & 0.100 & 0.100 & 0.091 & 0.108 \\
& 0.052 & 0.050 & 0.047 & 0.049 & 0.051 & 0.063 & 0.050 & 0.050 & 0.045 & 0.055 \\
& 0.023 & 0.023 & 0.023 & 0.007 & 0.010 & 0.013 & 0.010 & 0.010 & 0.008 & 0.012 \\
\hline \multirow{3}{*}{15,20} & 0.107 & 0.094 & 0.099 & 0.097 & 0.100 & 0.127 & 0.100 & 0.100 & 0.101 & 0.091 \\
& 0.053 & 0.046 & 0.046 & 0.049 & 0.051 & 0.067 & 0.050 & 0.050 & 0.049 & 0.043 \\
& 0.026 & 0.023 & 0.025 & 0.007 & 0.010 & 0.012 & 0.010 & 0.010 & 0.009 & 0.006 \\
\hline \multirow{3}{*}{20,20} & 0.104 & 0.102 & 0.100 & 0.097 & 0.104 & 0.123 & 0.100 & 0.100 & 0.084 & 0.090 \\
& 0.051 & 0.047 & 0.050 & 0.046 & 0.049 & 0.071 & 0.050 & 0.050 & 0.041 & 0.050 \\
& 0.025 & 0.023 & 0.024 & 0.007 & 0.009 & 0.019 & 0.010 & 0.010 & 0.006 & 0.011 \\
\hline
\end{tabular}

Table-1(b): Empirical Levels of Tests under Exponential Distribution based on 10, 000 replications.

\begin{tabular}{|c|c|c|c|c|c|c|c|c|c|c|}
\hline $\mathrm{n}_{1}, \mathrm{n}_{2}$ & Moods & $\begin{array}{c}\text { Ansari } \\
\text { Bradley }\end{array}$ & $\begin{array}{c}\text { Siegel } \\
\text { Tukey }\end{array}$ & Lepage & CVM & Kamat & $\begin{array}{c}\text { Wald } \\
\mathrm{W}\end{array}$ & $\begin{array}{c}\text { Wald } \\
\mathrm{R}\end{array}$ & Levene & $\mathrm{F}$ \\
\hline \multirow{3}{*}{5,10} & 0.108 & 0.108 & 0.108 & 0.090 & 0.105 & 0.111 & 0.235 & 0.119 & 0.096 & 0.222 \\
& 0.055 & 0.039 & 0.060 & 0.034 & 0.053 & 0.033 & 0.145 & 0.067 & 0.044 & 0.155 \\
& 0.029 & 0.021 & 0.023 & 0.003 & 0.009 & 0.007 & 0.052 & 0.017 & 0.007 & 0.074 \\
\hline \multirow{3}{*}{10,10} & 0.100 & 0.100 & 0.107 & 0.096 & 0.095 & 0.099 & 0.322 & 0.129 & 0.090 & 0.217 \\
& 0.053 & 0.038 & 0.052 & 0.040 & 0.045 & 0.055 & 0.236 & 0.075 & 0.042 & 0.158 \\
& 0.023 & 0.013 & 0.024 & 0.003 & 0.009 & 0.029 & 0.111 & 0.022 & 0.006 & 0.072 \\
\hline \multirow{3}{*}{10,15} & 0.104 & 0.095 & 0.098 & 0.101 & 0.100 & 0.196 & 0.323 & 0.125 & 0.098 & 0.216 \\
& 0.051 & 0.049 & 0.054 & 0.044 & 0.048 & 0.107 & 0.229 & 0.064 & 0.047 & 0.159 \\
& 0.026 & 0.022 & 0.024 & 0.006 & 0.010 & 0.020 & 0.091 & 0.021 & 0.008 & 0.082 \\
\hline \multirow{3}{*}{15,15} & 0.103 & 0.104 & 0.105 & 0.100 & 0.100 & 0.118 & 0.341 & 0.124 & 0.100 & 0.242 \\
& 0.051 & 0.054 & 0.048 & 0.048 & 0.049 & 0.055 & 0.259 & 0.067 & 0.047 & 0.184 \\
& 0.023 & 0.024 & 0.025 & 0.006 & 0.010 & 0.016 & 0.133 & 0.019 & 0.007 & 0.097 \\
\hline \multirow{3}{*}{15,20} & 0.099 & 0.099 & 0.105 & 0.102 & 0.102 & 0.126 & 0.343 & 0.115 & 0.101 & 0.210 \\
& 0.048 & 0.051 & 0.051 & 0.048 & 0.052 & 0.064 & 0.249 & 0.061 & 0.049 & 0.150 \\
& 0.025 & 0.027 & 0.028 & 0.006 & 0.009 & 0.012 & 0.113 & 0.014 & 0.007 & 0.069 \\
\hline \multirow{3}{*}{20,20} & 0.100 & 0.104 & 0.102 & 0.100 & 0.107 & 0.111 & 0.347 & 0.110 & 0.100 & 0.216 \\
& 0.050 & 0.053 & 0.054 & 0.050 & 0.052 & 0.063 & 0.262 & 0.057 & 0.047 & 0.169 \\
& 0.024 & 0.026 & 0.028 & 0.007 & 0.010 & 0.013 & 0.132 & 0.015 & 0.008 & 0.089 \\
\hline
\end{tabular}


Vol. 3, Issue 11, November 2016

Table-1(c): Empirical Levels of Tests under Double Exponential Distribution based on 10, 000 replications.

\begin{tabular}{|c|c|c|c|c|c|c|c|c|c|c|}
\hline $\mathrm{n}_{1}, \mathrm{n}_{2}$ & Moods & $\begin{array}{c}\text { Ansari } \\
\text { Bradley }\end{array}$ & $\begin{array}{c}\text { Siegel } \\
\text { Tukey }\end{array}$ & Lepage & CVM & Kamat & $\begin{array}{c}\text { Wald } \\
\mathrm{W}\end{array}$ & $\begin{array}{c}\text { Wald } \\
\mathrm{R}\end{array}$ & Levene & $\mathrm{F}$ \\
\hline \multirow{3}{*}{5,10} & 0.108 & 0.108 & 0.108 & 0.090 & 0.105 & 0.111 & 0.176 & 0.071 & 0.126 & 0.193 \\
& 0.055 & 0.039 & 0.060 & 0.034 & 0.053 & 0.033 & 0.104 & 0.031 & 0.062 & 0.126 \\
& 0.029 & 0.021 & 0.023 & 0.004 & 0.009 & 0.007 & 0.029 & 0.006 & 0.016 & 0.052 \\
\hline & 0.101 & 0.100 & 0.107 & 0.096 & 0.095 & 0.099 & 0.245 & 0.086 & 0.135 & 0.178 \\
10,10 & 0.053 & 0.038 & 0.052 & 0.041 & 0.045 & 0.055 & 0.163 & 0.041 & 0.070 & 0.121 \\
& 0.023 & 0.018 & 0.024 & 0.003 & 0.009 & 0.029 & 0.066 & 0.007 & 0.017 & 0.043 \\
\hline \multirow{3}{*}{10,15} & 0.104 & 0.095 & 0.098 & 0.102 & 0.100 & 0.196 & 0.243 & 0.086 & 0.139 & 0.188 \\
& 0.051 & 0.049 & 0.054 & 0.044 & 0.048 & 0.107 & 0.157 & 0.035 & 0.078 & 0.127 \\
& 0.026 & 0.022 & 0.024 & 0.007 & 0.010 & 0.020 & 0.052 & 0.005 & 0.015 & 0.054 \\
\hline \multirow{3}{*}{15,15} & 0.103 & 0.104 & 0.105 & 0.101 & 0.100 & 0.118 & 0.262 & 0.093 & 0.118 & 0.194 \\
& 0.051 & 0.054 & 0.048 & 0.048 & 0.049 & 0.055 & 0.177 & 0.041 & 0.058 & 0.134 \\
& 0.023 & 0.024 & 0.025 & 0.006 & 0.010 & 0.016 & 0.074 & 0.007 & 0.012 & 0.057 \\
\hline \multirow{3}{*}{15,20} & 0.099 & 0.099 & 0.105 & 0.102 & 0.102 & 0.126 & 0.257 & 0.089 & 0.123 & 0.172 \\
& 0.048 & 0.051 & 0.051 & 0.048 & 0.052 & 0.064 & 0.168 & 0.043 & 0.062 & 0.110 \\
& 0.025 & 0.027 & 0.028 & 0.006 & 0.009 & 0.012 & 0.060 & 0.006 & 0.013 & 0.041 \\
\hline \multirow{3}{*}{20,20} & 0.100 & 0.104 & 0.102 & 0.101 & 0.107 & 0.122 & 0.267 & 0.091 & 0.144 & 0.177 \\
& 0.050 & 0.053 & 0.054 & 0.050 & 0.052 & 0.067 & 0.182 & 0.044 & 0.079 & 0.129 \\
& 0.024 & 0.026 & 0.028 & 0.007 & 0.010 & 0.017 & 0.074 & 0.007 & 0.019 & 0.058 \\
\hline
\end{tabular}

Table-1(d): Empirical Levels of Tests under Logistic Distribution based on 10, 000 replications.

\begin{tabular}{|c|c|c|c|c|c|c|c|c|c|c|}
\hline $\mathrm{n}_{1}, \mathrm{n}_{2}$ & Moods & $\begin{array}{c}\text { Ansari } \\
\text { Bradley }\end{array}$ & $\begin{array}{c}\text { Siegel } \\
\text { Tukey }\end{array}$ & Lepage & CVM & Kamat & $\begin{array}{c}\text { Wald } \\
\mathrm{W}\end{array}$ & $\begin{array}{c}\text { Wald } \\
\mathrm{R}\end{array}$ & Levene & $\mathrm{F}$ \\
\hline \multirow{3}{*}{5,10} & 0.108 & 0.113 & 0.108 & 0.090 & 0.105 & 0.111 & 0.122 & 0.082 & 0.093 & 0.164 \\
& 0.053 & 0.038 & 0.060 & 0.035 & 0.053 & 0.033 & 0.068 & 0.037 & 0.041 & 0.091 \\
& 0.029 & 0.021 & 0.023 & 0.004 & 0.009 & 0.007 & 0.014 & 0.006 & 0.008 & 0.029 \\
\hline \multirow{3}{*}{10,10} & 0.101 & 0.102 & 0.107 & 0.096 & 0.095 & 0.099 & 0.163 & 0.093 & 0.095 & 0.134 \\
& 0.053 & 0.039 & 0.052 & 0.041 & 0.045 & 0.055 & 0.094 & 0.046 & 0.043 & 0.079 \\
& 0.023 & 0.019 & 0.024 & 0.003 & 0.009 & 0.029 & 0.026 & 0.008 & 0.007 & 0.021 \\
\hline \multirow{3}{*}{10,15} & 0.104 & 0.095 & 0.098 & 0.102 & 0.100 & 0.196 & 0.157 & 0.091 & 0.101 & 0.148 \\
& 0.051 & 0.050 & 0.054 & 0.044 & 0.048 & 0.107 & 0.089 & 0.041 & 0.047 & 0.087 \\
& 0.026 & 0.023 & 0.024 & 0.007 & 0.010 & 0.020 & 0.023 & 0.006 & 0.009 & 0.026 \\
\hline \multirow{3}{*}{15,15} & 0.103 & 0.100 & 0.105 & 0.101 & 0.100 & 0.118 & 0.167 & 0.097 & 0.102 & 0.147 \\
& 0.051 & 0.055 & 0.048 & 0.048 & 0.049 & 0.055 & 0.103 & 0.048 & 0.047 & 0.090 \\
& 0.023 & 0.025 & 0.025 & 0.006 & 0.010 & 0.016 & 0.031 & 0.010 & 0.008 & 0.028 \\
\hline \multirow{3}{*}{15,20} & 0.099 & 0.093 & 0.105 & 0.102 & 0.102 & 0.126 & 0.166 & 0.092 & 0.096 & 0.126 \\
& 0.048 & 0.048 & 0.051 & 0.048 & 0.052 & 0.064 & 0.093 & 0.047 & 0.047 & 0.071 \\
& 0.025 & 0.024 & 0.028 & 0.006 & 0.009 & 0.012 & 0.025 & 0.008 & 0.008 & 0.019 \\
\hline \multirow{3}{*}{20,20} & 0.101 & 0.103 & 0.102 & 0.101 & 0.107 & 0.122 & 0.173 & 0.094 & 0.101 & 0.130 \\
& 0.050 & 0.050 & 0.054 & 0.050 & 0.052 & 0.067 & 0.103 & 0.049 & 0.051 & 0.084 \\
& 0.024 & 0.024 & 0.028 & 0.007 & 0.010 & 0.0174 & 0.029 & 0.009 & 0.009 & 0.026 \\
\hline
\end{tabular}

Table-1(e): Empirical Levels of Tests under Cauchy Distribution based on 10, 000 replications.

\begin{tabular}{|c|c|c|c|c|c|c|c|c|c|c|}
\hline $\mathrm{n}_{1}, \mathrm{n}_{2}$ & Moods & $\begin{array}{c}\text { Ansari } \\
\text { Bradley }\end{array}$ & $\begin{array}{c}\text { Siegel } \\
\text { Tukey }\end{array}$ & Lepage & CVM & Kamat & $\begin{array}{c}\text { Wald } \\
\mathrm{W}\end{array}$ & $\begin{array}{c}\text { Wald } \\
\mathrm{R}\end{array}$ & Levene & $\mathrm{F}$ \\
\hline \multirow{3}{*}{5,10} & 0.110 & 0.113 & 0.113 & 0.087 & 0.096 & 0.110 & 0.549 & 0.033 & 0.069 & 0.283 \\
& 0.056 & 0.038 & 0.063 & 0.035 & 0.051 & 0.035 & 0.362 & 0.017 & 0.031 & 0.248 \\
& 0.028 & 0.021 & 0.022 & 0.003 & 0.011 & 0.007 & 0.251 & 0.004 & 0.005 & 0.193 \\
\hline & 0.101 & 0.102 & 0.109 & 0.091 & 0.092 & 0.107 & 0.677 & 0.046 & 0.053 & 0.378 \\
10,10 & 0.050 & 0.039 & 0.052 & 0.038 & 0.040 & 0.043 & 0.622 & 0.016 & 0.018 & 0.345 \\
& 0.023 & 0.019 & 0.027 & 0.005 & 0.008 & 0.020 & 0.504 & 0.002 & 0.002 & 0.280 \\
\hline & 0.105 & 0.095 & 0.099 & 0.097 & 0.099 & 0.178 & 0.695 & 0.039 & 0.005 & 0.338 \\
10,15 & 0.050 & 0.050 & 0.054 & 0.045 & 0.048 & 0.092 & 0.629 & 0.011 & 0.022 & 0.309 \\
\hline
\end{tabular}


International Advanced Research Journal in Science, Engineering and Technology

ISO 3297:2007 Certified

Vol. 3, Issue 11, November 2016

\begin{tabular}{|c|c|c|c|c|c|c|c|c|c|c|}
\hline & 0.026 & 0.023 & 0.024 & 0.006 & 0.011 & 0.017 & 0.480 & 0.001 & 0.002 & 0.260 \\
\hline & 0.103 & 0.100 & 0.103 & 0.102 & 0.099 & 0.120 & 0.729 & 0.036 & 0.057 & 0.407 \\
15,15 & 0.053 & 0.055 & 0.051 & 0.048 & 0.049 & 0.071 & 0.681 & 0.012 & 0.015 & 0.379 \\
& 0.025 & 0.025 & 0.025 & 0.007 & 0.010 & 0.010 & 0.584 & 0.001 & 0.001 & 0.324 \\
\hline \multirow{3}{*}{15,20} & 0.103 & 0.093 & 0.097 & 0.093 & 0.095 & 0.126 & 0.749 & 0.035 & 0.058 & 0.357 \\
& 0.050 & 0.048 & 0.048 & 0.043 & 0.045 & 0.064 & 0.697 & 0.011 & 0.022 & 0.329 \\
& 0.023 & 0.024 & 0.025 & 0.007 & 0.007 & 0.011 & 0.587 & 0.001 & 0.002 & 0.279 \\
\hline \multirow{3}{*}{20,20} & 0.104 & 0.103 & 0.099 & 0.099 & 0.105 & 0.143 & 0.771 & 0.035 & 0.061 & 0.397 \\
& 0.053 & 0.050 & 0.052 & 0.048 & 0.049 & 0.085 & 0.727 & 0.010 & 0.019 & 0.378 \\
& 0.026 & 0.024 & 0.025 & 0.007 & 0.008 & 0.022 & 0.642 & 0.000 & 0.001 & 0.334 \\
\hline
\end{tabular}

Table-2(a): Empirical Power of Tests under Normal Distribution

\begin{tabular}{|c|c|c|c|c|c|c|c|c|c|c|c|}
\hline $\mathrm{n}_{1}, \mathrm{n}_{2}$ & $\sigma_{1}, \sigma_{2}$ & Mood & $\begin{array}{c}\text { Ansari } \\
\text { Bradley }\end{array}$ & $\begin{array}{l}\text { Siegel } \\
\text { Tukey }\end{array}$ & Lepage & CVM & Kamat & $\begin{array}{c}\text { Wald } \\
-\mathrm{W}\end{array}$ & $\begin{array}{c}\text { Wald - } \\
\text { R }\end{array}$ & Levene & $\mathrm{F}$ \\
\hline \multirow[t]{4}{*}{$(5,10)$} & $(1.5,1)$ & 0.201 & 0.140 & 0.189 & 0.089 & 0.075 & 0.171 & 0.179 & 0.105 & 0.045 & 0.297 \\
\hline & $(2,1)$ & 0.356 & 0.248 & 0.326 & 0.176 & 0.105 & 0.322 & 0.365 & 0.178 & 0.112 & 0.544 \\
\hline & $(2.5,1)$ & 0.487 & 0.357 & 0.447 & 0.272 & 0.130 & 0.458 & 0.557 & 0.234 & 0.209 & 0.720 \\
\hline & $(3,1)$ & 0.584 & 0.448 & 0.543 & 0.357 & 0.152 & 0.566 & 0.715 & 0.274 & 0.317 & 0.828 \\
\hline \multirow[t]{4}{*}{$(10,10)$} & $(1.5,1)$ & 0.245 & 0.188 & 0.228 & 0.095 & 0.056 & 0.236 & 0.215 & 0.157 & 0.122 & 0.304 \\
\hline & $(2,1)$ & 0.476 & 0.385 & 0.431 & 0.209 & 0.074 & 0.476 & 0.521 & 0.337 & 0.309 & 0.628 \\
\hline & $(2.5,1)$ & 0.650 & 0.546 & 0.591 & 0.348 & 0.096 & 0.675 & 0.755 & 0.490 & 0.500 & 0.837 \\
\hline & $(3,1)$ & 0.758 & 0.658 & 0.701 & 0.475 & 0.118 & 0.801 & 0.887 & 0.596 & 0.648 & 0.933 \\
\hline \multirow[t]{4}{*}{$(10,15)$} & $(1.5,1)$ & 0.296 & 0.248 & 0.260 & 0.134 & 0.071 & 0.422 & 0.314 & 0.150 & 0.166 & .401 \\
\hline & $(2,1)$ & 0.561 & 0.486 & 0.502 & 0.299 & 0.105 & 0.699 & 0.665 & 0.347 & 0.432 & 0.745 \\
\hline & $(2.5,1)$ & 0.747 & 0.672 & 0.688 & 0.468 & 0.141 & 0.859 & 0.876 & 0.509 & 0.667 & 0.906 \\
\hline & $(3,1)$ & 0.846 & 0.794 & 0.803 & 0.602 & 0.178 & 0.929 & 0.958 & 0.614 & 0.808 & 0.968 \\
\hline \multirow[t]{4}{*}{$(15,15)$} & $(1.5,1)$ & 0.340 & 0.308 & 0.293 & 0.144 & 0.066 & 0.378 & 0.309 & 0.258 & 0.158 & 0.452 \\
\hline & $(2,1)$ & 0.653 & 0.596 & 0.576 & 0.357 & 0.104 & 0.703 & 0.717 & 0.565 & 0.467 & 0.823 \\
\hline & $(2.5,1)$ & 0.840 & 0.775 & 0.763 & 0.567 & 0.162 & 0.887 & 0.914 & 0.762 & 0.727 & 0.957 \\
\hline & $(3,1)$ & 0.923 & 0.878 & 0.866 & 0.714 & 0.222 & 0.959 & 0.977 & 0.858 & 0.871 & 0.992 \\
\hline \multirow[t]{4}{*}{$(15,20)$} & $(1.5,1)$ & 0.392 & 0.319 & 0.319 & 0.178 & 0.078 & 0.427 & 0.387 & 0.262 & 0.213 & 0.457 \\
\hline & $(2,1)$ & 0.723 & 0.646 & 0.645 & 0.439 & 0.137 & 0.763 & 0.800 & 0.584 & 0.580 & 0.843 \\
\hline & $(2.5,1)$ & 0.884 & 0.829 & 0.829 & 0.653 & 0.218 & 0.916 & 0.957 & 0.774 & 0.826 & 0.963 \\
\hline & $(3,1)$ & 0.952 & 0.920 & 0.919 & 0.791 & 0.306 & 0.970 & 0.992 & 0.862 & 0.933 & 0.992 \\
\hline \multirow[t]{4}{*}{$(20,20)$} & $(1.5,1)$ & 0.421 & 0.372 & 0.377 & 0.191 & 0.076 & 0.478 & 0.397 & 0.341 & 0.308 & 0.526 \\
\hline & $(2,1)$ & 0.781 & 0.709 & 0.712 & 0.483 & 0.139 & 0.825 & 0.836 & 0.729 & 0.719 & 0.900 \\
\hline & $(2.5,1)$ & 0.928 & 0.877 & 0.880 & 0.720 & 0.245 & 0.957 & 0.971 & 0.900 & 0.917 & 0.986 \\
\hline & $(3,1)$ & 0.976 & 0.946 & 0.947 & 0.857 & 0.371 & 0.988 & 0.995 & 0.954 & 0.979 & 0.998 \\
\hline
\end{tabular}

Table-2(b): Empirical power of Tests under Exponential Distribution

\begin{tabular}{|c|l|l|l|l|l|l|l|l|l|}
\hline $\mathrm{n}_{1}, \mathrm{n}_{2}$ & $\sigma_{1}, \sigma_{2}$ & Mood & Ansari Bradley & Siegel Tukey & Lepage & CVM & Kamat & Wald -R & Levene \\
\hline$(5,10)$ & $(1.5,1)$ & 0.083 & 0.053 & 0.080 & 0.050 & 0.093 & 0.061 & 0.100 & 0.047 \\
& $(2,1)$ & 0.104 & 0.054 & 0.082 & 0.084 & 0.166 & 0.079 & 0.137 & 0.080 \\
& $(2.5,1)$ & 0.120 & 0.051 & 0.075 & 0.128 & 0.250 & 0.088 & 0.178 & 0.126 \\
& $(3,1)$ & 0.129 & 0.042 & 0.065 & 0.172 & 0.335 & 0.091 & 0.208 & 0.173 \\
\hline$(10,10)$ & $(1.5,1)$ & .080 & 0.064 & 0.081 & 0.081 & 0.101 & 0.090 & 0.122 & 0.110 \\
& $(2,1)$ & 0.094 & 0.079 & 0.098 & 0.173 & 0.222 & 0.132 & 0.199 & 0.244 \\
& $(2.5,1)$ & 0.096 & 0.081 & 0.102 & 0.283 & 0.350 & 0.172 & 0.270 & 0.385 \\
& $(3,1)$ & 0.096 & 0.084 & 0.105 & 0.391 & 0.468 & 0.206 & 0.335 & 0.509 \\
\hline$(10,15)$ & $(1.5,1)$ & 0.093 & 0.074 & 0.081 & 0.093 & 0.123 & 0.163 & 0.106 & 0.105 \\
& $(2,1)$ & 0.127 & 0.087 & 0.095 & 0.202 & 0.268 & 0.217 & 0.188 & 0.246 \\
& $(2.5,1)$ & 0.150 & 0.086 & 0.094 & 0.330 & 0.432 & 0.256 & 0.267 & 0.401 \\
& $(3,1)$ & 0.166 & 0.079 & 0.087 & 0.462 & 0.573 & 0.283 & 0.333 & 0.544 \\
\hline$(15,15)$ & $(1.5,1)$ & 0.091 & 0.089 & 0.083 & 0.115 & 0.143 & 0.130 & 0.142 & 0.159 \\
& $(2,1)$ & 0.111 & 0.123 & 0.112 & 0.272 & 0.326 & 0.216 & 0.270 & 0.377 \\
& $(2.5,1)$ & 0.120 & 0.138 & 0.128 & 0.453 & 0.523 & 0.292 & 0.379 & 0.592 \\
\hline
\end{tabular}


International Advanced Research Journal in Science, Engineering and Technology

ISO 3297:2007 Certified

Vol. 3, Issue 11, November 2016

\begin{tabular}{|l|l|l|l|l|l|l|l|l|l|}
\hline & $(3,1)$ & 0.117 & 0.142 & 0.133 & 0.611 & 0.671 & 0.355 & 0.461 & 0.740 \\
\hline$(15,20)$ & $(1.5,1)$ & 0.101 & 0.094 & 0.094 & 0.128 & 0.160 & 0.137 & 0.125 & 0.160 \\
& $(2,1)$ & 0.142 & 0.115 & 0.114 & 0.308 & 0.377 & 0.217 & 0.251 & 0.408 \\
& $(2.5,1)$ & 0.174 & 0.119 & 0.119 & 0.511 & 0.591 & 0.286 & 0.369 & 0.627 \\
& $(3,1)$ & 0.198 & 0.116 & 0.116 & 0.680 & 0.750 & 0.344 & 0.458 & 0.780 \\
\hline$(20,20)$ & $(1.5,1)$ & 0.101 & 0.098 & 0.099 & 0.152 & 0.188 & 0.158 & 0.149 & 0.220 \\
& $(2,1)$ & 0.135 & 0.134 & 0.137 & 0.376 & 0.444 & 0.280 & 0.313 & 0.523 \\
& $(2.5,1)$ & 0.154 & 0.153 & 0.157 & 0.605 & 0.667 & 0.387 & 0.451 & 0.756 \\
& $(3,1)$ & 0.155 & 0.166 & 0.169 & 0.773 & 0.816 & 0.478 & 0.548 & 0.882 \\
\hline
\end{tabular}

Table-2c: Empirical power of Tests under Double Exponential Distribution

\begin{tabular}{|l|l|l|l|l|l|l|l|l|l|}
\hline $\mathrm{n}_{1}, \mathrm{n}_{2}$ & $\sigma_{1}, \sigma_{2}$ & Mood & Ansari Bradley & Siegel Tukey & Lepage & CVM & Kamat & Wald -R & Levene \\
\hline$(5$, & $(1.5,1)$ & 0.151 & 0.105 & 0.148 & 0.072 & 0.048 & 0.131 & 0.065 & 0.147 \\
$10)$ & $(2,1)$ & 0.261 & 0.184 & 0.248 & 0.138 & 0.057 & 0.238 & 0.108 & 0.249 \\
& $(2.5,1)$ & 0.367 & 0.262 & 0.339 & 0.224 & 0.070 & 0.326 & 0.148 & 0.346 \\
& $(3,1)$ & 0.458 & 0.331 & 0.421 & 0.317 & 0.084 & 0.411 & 0.178 & 0.428 \\
\hline$(10$, & $(1.5,1)$ & 0.178 & 0.141 & 0.171 & 0.074 & 0.069 & 0.168 & 0.084 & 0.145 \\
$10)$ & $(2,1)$ & 0.338 & 0.277 & 0.321 & 0.124 & 0.089 & 0.315 & 0.171 & 0.272 \\
& $(2.5,1)$ & 0.487 & 0.405 & 0.455 & 0.187 & 0.110 & 0.456 & 0.255 & 0.396 \\
& $(3,1)$ & 0.609 & 0.519 & 0.565 & 0.249 & 0.126 & 0.577 & 0.321 & 0.505 \\
\hline$(10$, & $(1.5,1)$ & 0.213 & 0.186 & 0.196 & 0.101 & 0.061 & 0.303 & 0.071 & 0.189 \\
$15)$ & $(2,1)$ & 0.411 & 0.361 & 0.373 & 0.199 & 0.083 & 0.513 & 0.162 & 0.371 \\
& $(2.5,1)$ & 0.583 & 0.512 & 0.531 & 0.323 & 0.109 & 0.676 & 0.253 & 0.539 \\
& $(3,1)$ & 0.705 & 0.636 & 0.651 & 0.439 & 0.137 & 0.788 & 0.329 & 0.667 \\
\hline$(15$, & $(1.5,1)$ & 0.239 & 0.234 & 0.218 & 0.107 & 0.060 & 0.239 & 0.131 & 0.178 \\
$15)$ & $(2,1)$ & 0.472 & 0.451 & 0.433 & 0.235 & 0.082 & 0.449 & 0.291 & 0.389 \\
& $(2.5,1)$ & 0.663 & 0.625 & 0.611 & 0.382 & 0.110 & 0.648 & 0.427 & 0.568 \\
& $(3,1)$ & 0.792 & 0.749 & 0.734 & 0.517 & 0.144 & 0.788 & 0.534 & 0.707 \\
\hline$(15$, & $(1.5,1)$ & 0.265 & 0.248 & 0.248 & 0.123 & 0.068 & 0.274 & 0.111 & 0.219 \\
$20)$ & $(2,1)$ & 0.527 & 0.492 & 0.492 & 0.280 & 0.100 & 0.515 & 0.266 & 0.466 \\
& $(2.5,1)$ & 0.730 & 0.687 & 0.687 & 0.459 & 0.142 & 0.700 & 0.419 & 0.664 \\
& $(3,1)$ & 0.849 & 0.805 & 0.805 & 0.607 & 0.192 & 0.824 & 0.534 & 0.798 \\
\hline$(20$, & $(1.5,1)$ & 0.289 & 0.270 & 0.273 & 0.132 & 0.065 & 0.304 & 0.151 & 0.236 \\
$20)$ & $(2,1)$ & 0.589 & 0.548 & 0.553 & 0.318 & 0.101 & 0.578 & 0.373 & 0.508 \\
& $(2.5,1)$ & 0.795 & 0.742 & 0.746 & 0.518 & 0.151 & 0.768 & 0.561 & 0.716 \\
& $(3,1)$ & 0.901 & 0.858 & 0.862 & 0.682 & 0.214 & 0.877 & 0.680 & 0.842 \\
\hline
\end{tabular}

Table-2d: Empirical power of Tests under Cauchy distribution

\begin{tabular}{|l|l|l|l|l|l|l|l|l|l|}
\hline $\mathrm{n}_{1}, \mathrm{n}_{2}$ & $\sigma_{1}, \sigma_{2}$ & Mood & Ansari Bradley & Siegel Tukey & Lepage & CVM & Kamat & Wald-R & Levene \\
\hline & $(1.5,1)$ & 0.117 & 0.093 & 0.133 & 0.059 & 0.063 & 0.085 & 0.028 & 0.063 \\
$(5,10)$ & $(2,1)$ & 0.183 & 0.149 & 0.201 & 0.089 & 0.075 & 0.137 & 0.038 & 0.103 \\
& $(2.5,1)$ & 0.250 & 0.205 & 0.275 & 0.131 & 0.090 & 0.180 & 0.047 & 0.141 \\
& $(3,1)$ & 0.311 & 0.255 & 0.331 & 0.167 & 0.102 & 0.219 & 0.057 & 0.181 \\
\hline$(10$, & $(1.5,1)$ & 0.135 & 0.115 & 0.141 & 0.057 & 0.042 & 0.110 & 0.023 & 0.033 \\
$10)$ & $(2,1)$ & 0.229 & 0.206 & 0.242 & 0.103 & 0.051 & 0.191 & 0.037 & 0.059 \\
& $(2.5,1)$ & 0.323 & 0.292 & 0.336 & 0.157 & 0.060 & 0.268 & 0.049 & 0.092 \\
& $(3,1)$ & 0.412 & 0.378 & 0.424 & 0.213 & 0.068 & 0.336 & 0.061 & 0.121 \\
\hline$(10$, & $(1.5,1)$ & 0.155 & 0.145 & 0.155 & 0.076 & 0.057 & 0.178 & 0.016 & 0.054 \\
$15)$ & $(2,1)$ & 0.276 & 0.267 & 0.279 & 0.139 & 0.072 & 0.265 & 0.026 & 0.101 \\
& $(2.5,1)$ & 0.395 & 0.384 & 0.395 & 0.216 & 0.087 & 0.344 & 0.037 & 0.151 \\
& $(3,1)$ & 0.496 & 0.481 & 0.494 & 0.298 & 0.103 & 0.414 & 0.049 & 0.205 \\
\hline$(15$, & $(1.5,1)$ & 0.177 & 0.176 & 0.166 & 0.084 & 0.057 & 0.145 & 0.019 & 0.035 \\
$15)$ & $(2,1)$ & 0.319 & 0.328 & 0.312 & 0.169 & 0.069 & 0.249 & 0.035 & 0.076 \\
& $(2.5,1)$ & 0.459 & 0.466 & 0.448 & 0.261 & 0.087 & 0.326 & 0.050 & 0.120 \\
& $(3,1)$ & 0.570 & 0.586 & 0.568 & 0.361 & 0.108 & 0.405 & 0.064 & 0.161 \\
\hline$(15$, & $(1.5,1)$ & 0.190 & 0.181 & 0.181 & 0.096 & 0.061 & 0.145 & 0.017 & 0.056 \\
\hline
\end{tabular}


International Advanced Research Journal in Science, Engineering and Technology

ISO 3297:2007 Certified

Vol. 3, Issue 11, November 2016

\begin{tabular}{|l|l|l|l|l|l|l|l|l|l|}
\hline 20$)$ & $(2,1)$ & 0.360 & 0.356 & 0.356 & 0.196 & 0.081 & 0.240 & 0.028 & 0.108 \\
& $(2.5,1)$ & .516 & 0.515 & 0.513 & 0.313 & 0.107 & 0.323 & 0.039 & 0.166 \\
& $(3,1)$ & 0.641 & 0.634 & 0.633 & 0.428 & 0.139 & 0.404 & 0.054 & 0.228 \\
\hline$(20$, & $(1.5,1)$ & 0.206 & 0.208 & 0.212 & 0.105 & 0.060 & 0.194 & 0.020 & 0.044 \\
$20)$ & $(2,1)$ & 0.399 & 0.405 & 0.412 & 0.216 & 0.082 & 0.294 & 0.034 & 0.097 \\
& $(2.5,1)$ & 0.561 & 0.573 & 0.579 & 0.356 & 0.116 & 0.390 & 0.051 & 0.147 \\
& $(3,1)$ & 0.692 & 0.702 & 0.708 & 0.485 & 0.153 & 0.472 & 0.063 & 0.200 \\
\hline
\end{tabular}

Table-2e Empirical power of Tests under Logistic Distribution

\begin{tabular}{|c|l|l|l|l|l|l|l|l|l|l|}
\hline $\mathrm{n}_{1}, \mathrm{n}_{2}$ & $\sigma_{1}, \sigma_{2}$ & Mood & $\begin{array}{c}\text { Ansari } \\
\text { Bradley }\end{array}$ & $\begin{array}{c}\text { Siegel } \\
\text { Tukey }\end{array}$ & Lepage & CVM & Kamat & Wald -W & Wald - R & Levene \\
\hline \multirow{3}{*}{$(5,10)$} & $(1.5,1)$ & 0.180 & 0.121 & 0.171 & 0.084 & 0.075 & 0.158 & 0.203 & 0.084 & 0.146 \\
& $(2,1)$ & 0.325 & 0.223 & 0.299 & 0.156 & 0.103 & 0.296 & 0.387 & 0.145 & 0.313 \\
& $(2.5,1)$ & 0.451 & 0.326 & 0.412 & 0.243 & 0.124 & 0.410 & 0.560 & 0.197 & 0.463 \\
& $(3,1)$ & 0.553 & 0.416 & 0.507 & 0.325 & 0.144 & 0.516 & 0.698 & 0.235 & 0.585 \\
\hline \multirow{5}{*}{$(10,10)$} & $(1.5,1)$ & 0.219 & 0.173 & 0.207 & 0.085 & 0.049 & 0.209 & 0.247 & 0.116 & 0.145 \\
& $(2,1)$ & 0.425 & 0.346 & 0.395 & 0.181 & 0.064 & 0.899 & 0.515 & 0.256 & 0.334 \\
& $(2.5,1)$ & 0.605 & 0.506 & 0.556 & 0.308 & 0.083 & 0.584 & 0.723 & 0.383 & 0.529 \\
& $(3,1)$ & 0.726 & 0.627 & 0.671 & 0.423 & 0.101 & 0.718 & 0.851 & 0.475 & 0.677 \\
\hline \multirow{5}{*}{$(10,15)$} & $(1.5,1)$ & 0.262 & 0.224 & 0.238 & 0.123 & 0.066 & 0.367 & 0.346 & 0.109 & 0.212 \\
& $(2,1)$ & 0.512 & 0.447 & 0.461 & 0.264 & 0.098 & 0.624 & 0.646 & 0.253 & 0.476 \\
& $(2.5,1)$ & 0.701 & 0.625 & 0.639 & 0.425 & 0.135 & 0.798 & 0.838 & 0.391 & 0.689 \\
& $(3,1)$ & 0.815 & 0.747 & 0.761 & 0.557 & 0.169 & 0.892 & 0.933 & 0.489 & 0.827 \\
\hline \multirow{5}{*}{$(15,15)$} & & & & & & & & & & \\
& $(1.5,1)$ & 0.297 & 0.283 & 0.276 & 0.132 & 0.062 & 0.300 & 0.344 & 0.191 & 0.217 \\
& $(2,1)$ & 0.585 & 0.553 & 0.535 & 0.313 & 0.096 & 0.588 & 0.682 & 0.433 & 0.517 \\
& $(2.5,1)$ & 0.787 & 0.736 & 0.722 & 0.507 & 0.142 & 0.789 & 0.877 & 0.621 & 0.750 \\
& $(3,1)$ & 0.891 & 0.846 & 0.836 & 0.663 & 0.196 & 0.897 & 0.955 & 0.731 & 0.882 \\
\hline \multirow{5}{*}{$(15,20)$} & & & & & & & & & & \\
& $(1.5,1)$ & 0.330 & 0.310 & 0.309 & 0.154 & 0.074 & 0.342 & 0.421 & 0.173 & 0.270 \\
& $(2,1)$ & 0.651 & 0.610 & 0.611 & 0.380 & 0.122 & 0.639 & 0.772 & 0.424 & 0.617 \\
& $(2.5,1)$ & 0.844 & 0.796 & 0.795 & 0.590 & 0.186 & 0.828 & 0.929 & 0.620 & 0.842 \\
& $(3,1)$ & 0.927 & 0.894 & 0.893 & 0.746 & 0.264 & 0.919 & 0.979 & 0.737 & 0.938 \\
\hline \multirow{5}{*}{$(20,20)$} & & & & & & & & & & \\
& $(1.5,1)$ & 0.367 & 0.335 & 0.341 & 0.168 & 0.070 & 0.374 & 0.423 & 0.237 & 0.280 \\
& $(2,1)$ & 0.725 & 0.663 & 0.668 & 0.428 & 0.127 & 0.718 & 0.798 & 0.561 & 0.665 \\
& $(2.5,1)$ & 0.897 & 0.849 & 0.851 & 0.667 & 0.209 & 0.890 & 0.943 & 0.766 & 0.882 \\
& $(3,1)$ & 0.962 & 0.931 & 0.933 & 0.817 & 0.317 & 0.961 & 0.986 & 0.860 & 0.962 \\
\hline
\end{tabular}

Table-3 Simulated Critical Values for Wald - W Test and Wald-R Test

\begin{tabular}{|c|c|c|c|c|c|c|c|}
\hline \multicolumn{2}{|c|}{ Sample Size } & \multicolumn{3}{|c|}{ Wald Test $(\mathrm{W})$} & \multicolumn{3}{|c|}{ Wald Test (R) } \\
\hline 5 & 10 & 3.1892 & 4.0945 & 5.2790 & 8.1734 & 12.5656 & 32.9237 \\
\hline 10 & 10 & 2.5581 & 3.1708 & 4.2124 & 4.7939 & 6.5617 & 11.8474 \\
\hline 10 & 15 & 2.7347 & 3.5266 & 5.1450 & 4.4941 & 6.4015 & 11.1498 \\
\hline 15 & 15 & 2.6283 & 3.3485 & 4.7258 & 3.8587 & 5.2409 & 8.6041 \\
\hline 15 & 20 & 2.6942 & 3.6037 & 5.3406 & 3.7735 & 5.2283 & 9.0331 \\
\hline 20 & 20 & 2.6555 & 3.5032 & 5.1984 & 3.5842 & 4.9308 & 8.2530 \\
\hline 20 & 25 & 2.6874 & 3.5553 & 5.4277 & 3.4807 & 4.8885 & 8.0067 \\
\hline 25 & 25 & 2.6784 & 3.6216 & 5.3316 & 3.3674 & 4.6840 & 7.8428 \\
\hline 30 & 30 & 2.6317 & 3.6175 & 5.4279 & 3.1931 & 4.4862 & 7.5974 \\
\hline
\end{tabular}


Fig : 1

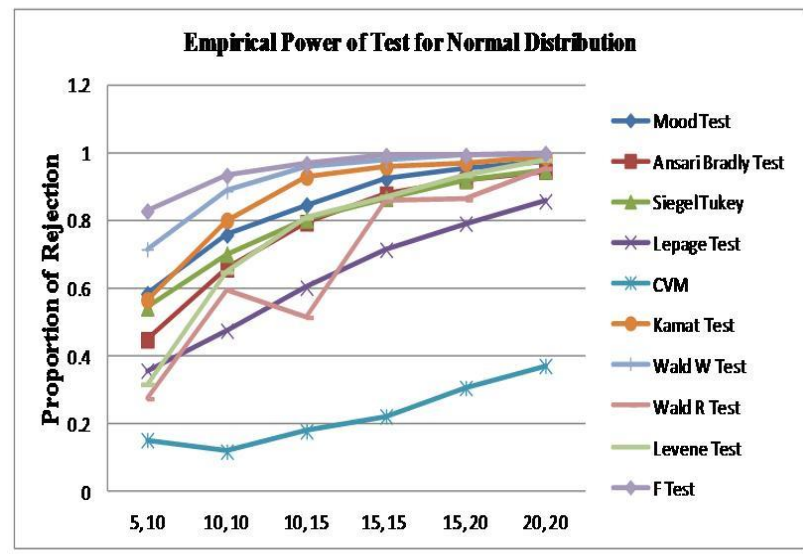

Sample Size

Fig : 3

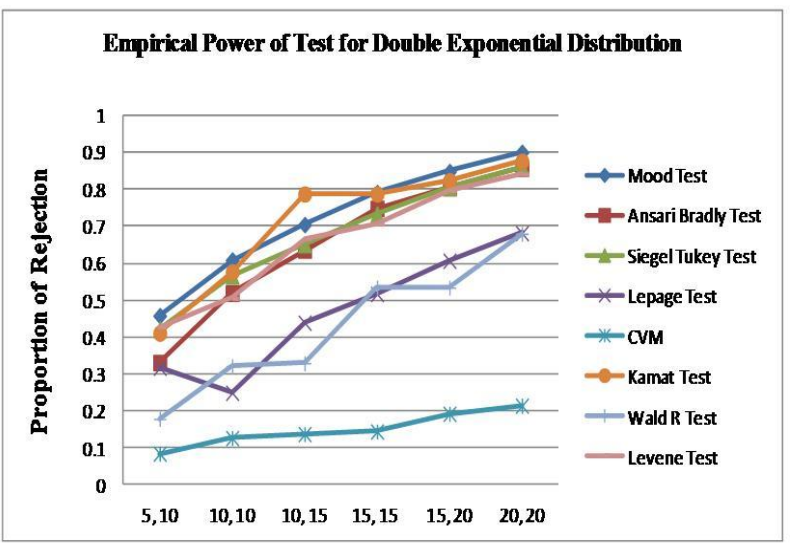

Sample Size

\section{DISCUSSION}

Tables and graphs above show the empirical level and In Cauchy distribution, Empirical power of Ansaripower of test statistics under different distributions, Bradley, Mood and Siegel-Tukey are found to be more various combinations of scale parameters and sample sizes.

As we observed in normal distribution, F-test gives the highest power and simultaneously the performance of Wald-W, Kamat, Mood, Ansari-Bradley, Siegel-Tukey, Lepage and Levene test are also quite good although slightly less than the F-test. Under this distribution CVM test shows the least power of all.

In exponential distribution, CVM test gives the more power against the small sample sizes and Levene test gives the highest power for the large sample sizes. Empirical power of Lepage also lies neck to neck with these two tests. Power of Kamat and Wald are less than these three tests but higher than Siegel-Tukey, Ansari-Bradley and Mood test. The last three tests give the almost similar power but less other tests.

Under double exponential distribution, except CVM and Wald tests, the performance of Mood, Ansari-Bradley, Siegel-Tukey, Kamat and Levene tests are more or less similar in power, so we may choose any one of them.
Fig : 2

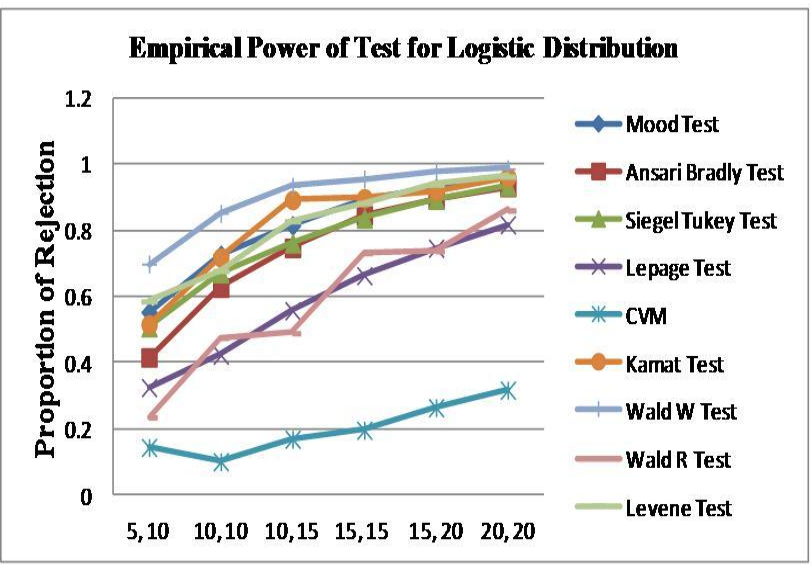

Sample Size

Fig : 4

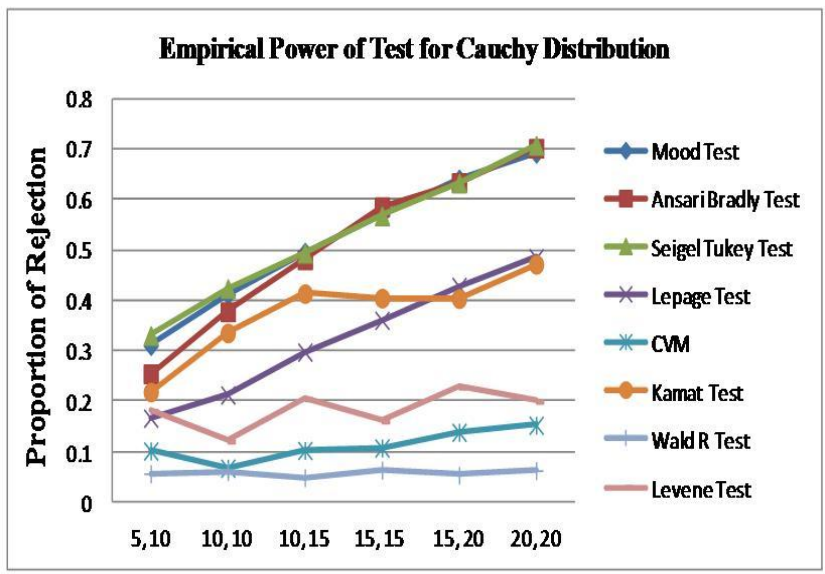


Ansari-Bradley and Siegel-Tukey are found to be powerful in Cauchy distribution. Finally, Wald-W test found to be more powerful in logistic distribution. Accordingly one may choose the tests statistics for testing equality of variance of two populations.

\section{REFERENCES}

[1] Ansari, A.R. and Bradley, R. A. (1960): Rank-sum tests for dispersion. Ann. Math. Stat., 31, 1174-1189.

[2] Allingham, D. and Rayner, J. (2011): Two-sample testing for equality of variances. ASEARC, Conferrence paper.

[3] Bunning, H. and Thadewald, T. (2000): An Adaptive two-sample location-scale test of Lepage type for symmetric distributions. Jour. Statist Comput. Simul., 65, 287-310.

[4] Burr, E. J. (1963): Distribution of the Two-Sample Cramer-VonMises Criterion for small equal samples. Ann. Math. Stat., 34(1), 95-101

[5] Hall, P. and Padmanabhan, A. R. (1997): Adaptive Inference for the two-sample scale problem. Technometrics, 39(4), 412-422.

[6] Hollander, M. and Wolfe, D.A. (1999): Nonparametric statistical methods, $2^{\text {nd }}$ Edn. New York, Wiley.

[7] Kamat, A.R. (1956): A two-sample distribution-free test. Biometrika, 43,377-387.

[8] Lehmann, E. L. (1951): Consistency and unbiasedness of certain nonparametric test. Ann. Math. Stat., 22, $165-179$.

[9] Lepage, Y. (1971): A combination of Wilcoxon's and Ansari Bradley's statistics. Biometrika, 58, 213-217.

[10] Levene, H. (1960): Robust tests for equality of variances. In: Contributions to Probability and Statistics, Ed, Olkin, I., Standford University Press, Palo Alto, 278-292.

[11] Mood, A. M.(1954) : On the asymptotic efficiency of certain nonparametric two-sample tests. Ann. Math. Stat., 25(3), 514-522.

[12] Mielke, P. W. Jr. (1972): Asymptotic behaviour of two-sample tests based on powers of ranks for detecting scale and location alternatives. Jour. Amer. Stat. Assoc., 67, 850-854.

[13] Miller, R. G. (1968): Jackknifing variances. Ann. Math. Stat., 39, 567-582.

[14] Perng, S.K. and Littell, R.C. (1976): A test of equality of two normal population means and variances. Jour. Amer. Stat. Assoc., 71, $968-971$.

[15] Padmanabhan, A. R., Othman, A.R. and Yin, T. S. (2011): A robust test based on bootstrapping for the two-sample problem. Sains Malaysiana, 40(5), 521-525.

[16] Rosenbaum, S. (1953): Tables for a nonparametric tests of dispersion. Ann. Math. Stat., 24(3), 663-668

[17] Sukhatme, B. V. (1957): On certain two sample nonparametric tests for variances. Ann. Math. Stat. 28(1), 188-194.

[18] Seigel, S. And Tukey, J. W. (1960): A non-parametric sum of ranks procedure for relative spread in unpaired samples. Jour. Amer. Stat. Assoc. 55, 429-445. 